\title{
Perioperative Management of Multiple Intracranial Nail Gun Injuries
}

\author{
Matthew B Ellison, MD*, Jeremy Hensley, MD, Roy E Henrickson, MD, Jeffrey Puette, MD, \\ Jason Shepherd, MD, Daniel Sloyer, MD and Pavithra R Ellison, MD
}

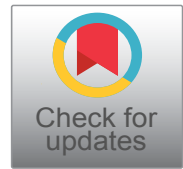

Department of Anesthesiology, West Virginia University, USA

*Corresponding author: Matthew B Ellison, MD, Department of Anesthesiology, West Virginia University School of Medicine, PO Box 8255, 1 Medical Center Drive, Morgantown, WV 26506, USA, Tel: 304-285-1931, Fax: 304-598-4930

\begin{abstract}
A 51-year-old male presented to the emergency department with four self-inflicted nail gun wounds. Computed tomography angiography demonstrated three nails penetrating the frontal bone toward the falx cerebri causing small subdural hematomas. The fourth nail penetrated the parietal bone posteriorly, ending very close to the vein of Galen. Despite the ominous-appearing imaging, the patient was neurologically intact and cooperative to the point that consideration was given to the possibility of removal of the nails under local anesthesia. Due to the number of nails and proximity of the fourth nail to a major intracranial blood vessel, a general anesthetic was performed observing the usual considerations for a traumatic brain injury. The nails were successfully removed through four small craniotomies and the patient had no complications post-operatively.

Intracranial nail gun injuries are unique among traumatic brain injuries in that these patients may present with a normal neurologic exam. When a victim of nail gun injury is neurologically intact, awake extraction under local anesthesia may be advantageous because it offers continuous neurologic surveillance to detect pseudoaneurysm rupture or new hematoma. Important considerations for intracranial foreign body extraction under local anesthesia include unanticipated voluntary or seizure induced head movement that may result in catastrophic injury. In addition, awake patients may not tolerate multiple local anesthetic injections or long operative time. The presence of nails in high risk locations or when vascular compromise is suspected are best managed with general anesthesia.
\end{abstract}

\section{Keywords}

Penetrating brain injury, Nail gun, Traumatic brain injury, Self-inflicted injury

\begin{abstract}
Abbreviations
TBI: Traumatic Brain Injury; ICP: Intracranial Pressure; PBI: Penetrating Brain Injury; GCS: Glasgow Coma Score; CT: Computerized Tomography; OR: Operating Room; RSI: Rapid Sequence Induction; ED: Emergency Department; IV: Intravenous; ICU: Intensive Care Unit; ATLS: Advanced Trauma Life Support; CPP: Cerebral Perfusion Pressure; MAP: Mean Arterial Pressure
\end{abstract}

\section{Introduction}

Traumatic brain injury (TBI) is a serious and common public health issue not only in the United States, but in the entire world. Almost 2 million people endure a TBI in the United States every year, leading to over fifty thousand deaths [1]. According to the National Center for Injury Prevention and Control, TBI is a major contributor in nearly one-third of all trauma mortalities [1]. TBI is often polytraumatic in nature, meaning that it is accompanied by other traumatic injuries. The cause of death from TBI often occurs secondary to related insults including hypoxia, hypotension, and severely increased intracranial pressure (ICP) from edema or hematoma [1]. A penetrating brain injury (PBI) is a specific category of $\mathrm{TBI}$ in which an object penetrates the skull. The object may be high velocity as in the case of a gun bullet or low velocity, such as a knife, nail, or other sharp object. The pneumatic nail gun, which is commonly used in the construction industry and is easily accessible, was first introducing in the late 1950's [2]. Since its inception and widespread adoption of this tool, reports

Citation: Ellison MB, Hensley J, Henrickson RE, Puette J, Shepherd J, et al. (2019) Perioperative Management of Multiple Intracranial Nail Gun Injuries. Int J Anesthetic Anesthesiol 7:100. doi. org/10.23937/2377-4630/1410100

Accepted: January 02, 2020: Published: January 04, 2020

Copyright: (C) 2019 Ellison MB, et al. This is an open-access article distributed under the terms of the Creative Commons Attribution License, which permits unrestricted use, distribution, and reproduction in any medium, provided the original author and source are credited. 
of the traumatic nail gun PBI's in the literature have steadily increased. A Centers for Disease Control and Prevention (CDC) report from 2005 stated that nail gun injuries seen in the emergency departments in the United States have increased 300\% from the early 1990's until the mid-2000's, with approximately 37,000 patients treated from 2001-2005 [3]. These types of PBI's present a unique set of challenges and highlight the importance of multi-disciplinary collaboration between the medical specialties.

\section{Case Description}

A 51-year-old male was admitted to the emergen- cy department several hours after sustaining a self-inflicted nail gun injury. The patient had no significant past medical history and had attempted suicide by placing four $7.5 \mathrm{~cm}$ nails through his calvarium with a pneumatic nail gun. The four nails entered the skull at the following locations: Left frontal midline, left frontal lateral, right frontal lateral, and left temporal (Figure 1). The patient was completely neurologically intact upon presentation with no cranial nerve deficits and a Glasgow Coma Score (GCS) of 15. In fact, the patient had arrived in the emergency department (ED) as a walk-in. After his initial assessment in the emergency department detected no additional inju-

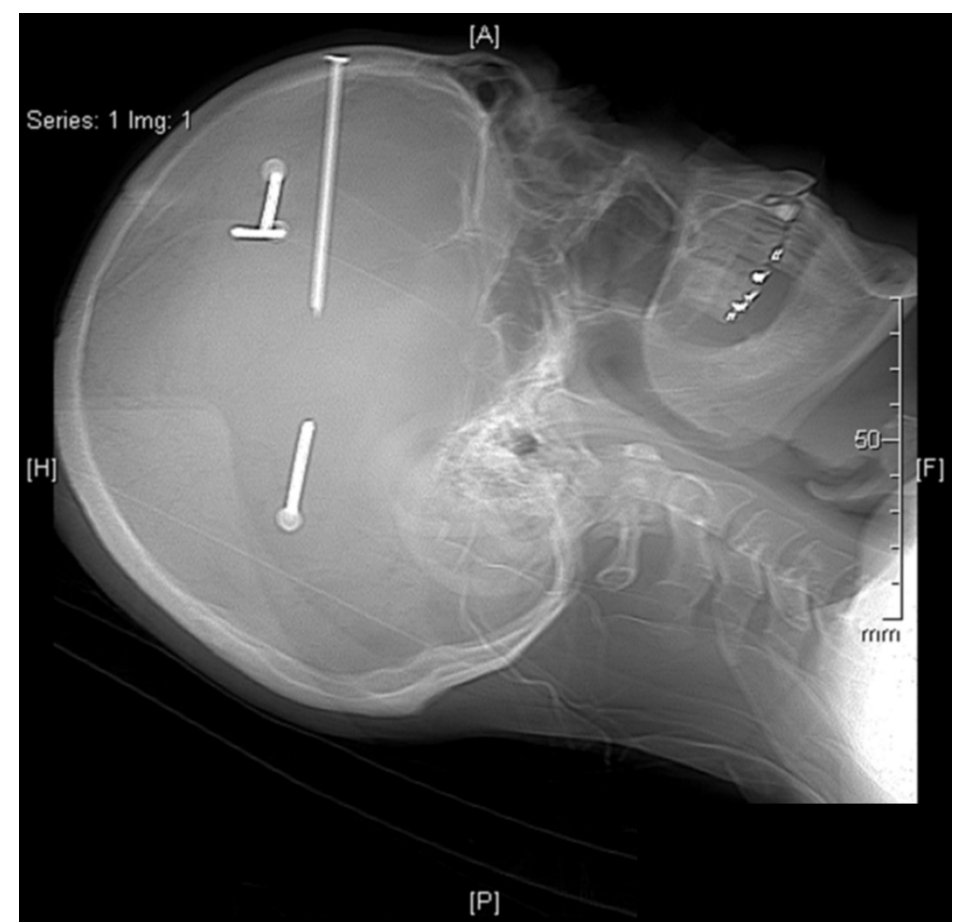

Figure 1: Sagittal x-ray showing the presence of four $7.5 \mathrm{~cm}$ nails within the cranial cavity.

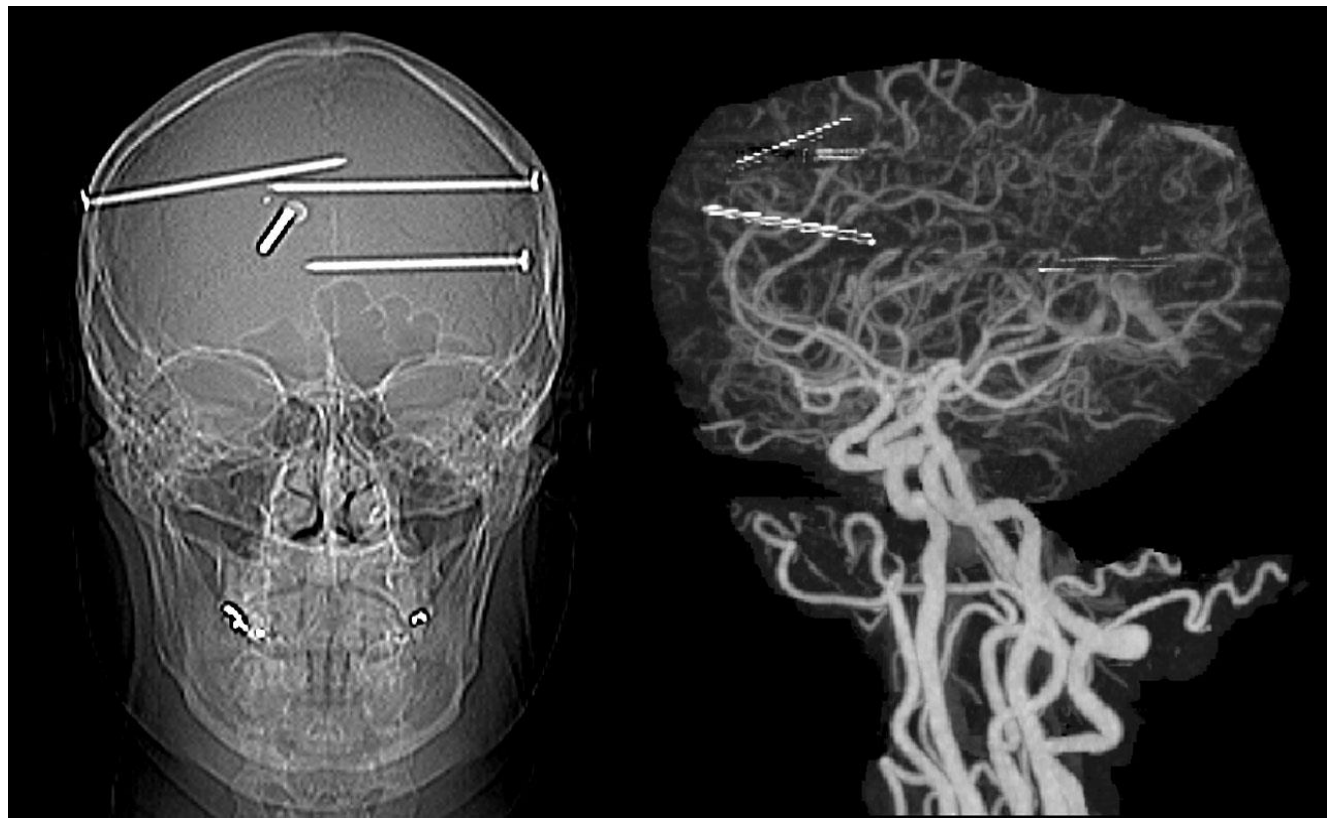

Figure 2: Image on the left is coronal $x$-ray view demonstrating the four nails within the cranium. The image on the right is computed tomography $(\mathrm{CT})$ angiography showing the nails in relation to the intracranial vasculature. 
ries, the patient was sent immediately to radiology for imaging. The initial computerized tomography (CT) scan demonstrated a small intracranial hemorrhage in the form of a subdural hematoma located bilaterally along the anterior falx cerebri. In addition, there was a small subarachnoid hemorrhage over both frontal lobes but no midline shift. A follow-up CT angiogram failed to show the presence of a pseudoaneurysm (Figure 2). Laboratory values on admission were also within normal reference ranges. The patient was given antibiotics and a tetanus booster in the ED.

After consultation between the neurosurgical service and anesthesiology, the decision was made to remove the nails in the operating room (OR) under general anesthesia rather than under with local anesthesia. The patient was taken to the OR and standard monitors were applied. A preoperative radial arterial line was placed prior to induction utilizing $1 \%$ lidocaine for local anesthesia. Cross-matched packed red blood cells were brought to the OR. The patient was placed in reverse Trendelenburg position in order to minimize ICP increase with intubation. A rapid sequence induction (RSI) was performed because of the patient's recent food consumption utilizing lidocaine, fentanyl, propofol, and rocuronium. On induction, care was taken in order to minimize increases in ICP while simultaneously maintaining blood pressure and therefore, cerebral perfusion pressure. An additional large bore intravenous (IV) catheter (14 gauge) was placed after induction, in addition to the patient's current 18 gauge IV catheter. The operating room table was turned 180 degrees and the patient was shaved and prepped. Three small craniectomies were performed through which three of the four 7.5 $\mathrm{cm}$ nails were removed. According to the imaging, the fourth nail was projecting near the vein of Galen and in the straight sinus and there was concern for possible disruption of this large vascular structure. This nail was removed without incident and the injuries were closed without cranioplasty. The wounds were irrigated, and Floseal (a gelatin-thrombin matrix sealant [Baxter, Hayward, CA, USA]) and Avitene (microfibrillar collagen hemostat [Warwick, RI, USA]) were used for hemostasis prior to closure. After completion of the surgery, the patient was left intubated in order to obtain a post-operative CT scan. No acute hemorrhage was appreciated and the patient was taken to the neurosurgical intensive care unit (ICU). He was extubated within thirty minutes of completion of the surgery and was neurologically intact. The patient remained stable overnight in the ICU and was transferred to the floor the next day. The remainder of his hospitalization was uneventful and he was discharged home on post-operative day two.

\section{Discussion}

The initial approach to any traumatic brain injury
(TBI) patient is still centered on the Advanced Trauma Life Support (ATLS) guidelines from the American College of Surgeons where the emphasis is placed on airway, breathing, and circulation with a subsequent neurologic exam and exposure with the goal of hypothermia prevention [4]. TBI is frequently associated with other traumatic injury including abdominal, orthopedic, and thoracic. Many of these TBI patients will have cervical spine injuries but this may be less common with self-inflicted PBI. Patients who present to the ED or the OR with a TBI are classified according to their GCS score: A GCS of 13-15 is labeled as a mild head injury, a GCS of 9-12 is a moderate head injury, and a GCS below 9 is classified as severe and corresponds with a $35 \%$ mortality [5]. If the GCS is < 9 or if a CT scan demonstrates a midline shift $>5 \mathrm{~mm}$ or absent ventricles, then immediate intubation for airway protection is likely indicated. The patient's ICP may be substantially increased, which could significantly impair ventilation. In patients with an acute TBI and who are suspected to have increased ICP, an external ventricular drain may be placed in order to accurately monitor the ICP. Surgical intervention is typically indicated in TBI for patients who have a midline shift $>5$ $\mathrm{mm}$, basal cistern compression, refractory elevation of ICP, acutely expanding intracranial hemorrhage, depressed skull fracture, and retained foreign body such as a nail $[6,7]$. Specifically in cases of penetrating head injury, indications for surgery include retained foreign object, presence of dural defects, displaced bone fractures, intracranial hematoma, and direct vascular injury [8].

Overall management goals for these patients emphasize the avoidance of significant secondary injuries, which are often from hypotension and hypoxia. Throughout the perioperative period, the patient's cerebral perfusion pressure (CPP) should be maintained, in order to achieve adequate cerebral blood flow. CPP can be improved by lowering the ICP, raising the MAP, or a combination of both. Vasopressor agents are frequently recommended to maintain or raise the MAP, along with intravenous fluids or blood if the patient is hypovolemic from other injuries. Hypertension should also be avoided to prevent exacerbation of hemorrhage and increased ICP. The ideal anesthetic induction should decrease cerebral metabolic rate and ICP while maintaining MAP and therefore, CPP. Propofol is an ideal induction agent as it decreases cerebral metabolic, does not increase $I C P$, raises the seizure threshold, and is neuroprotective in many models of neuronal injury [9]. However, caution must be exercised with dosing, as large doses may decrease MAP and CPP. In addition, general anesthesia with muscle relaxation is preferred, especially when the foreign body is in close proximity to major vascular structures and patient movement could be disastrous. Many of these patients are at 
high risk for aspiration and a rapid sequence induction may be considered with either succinylcholine or rocuronium. A small transient rise in ICP has been reported with the use of succinylcholine, but it has been safely used in clinical practice and may be used if the need to rapidly secure the airway outweighs the potential risk. Higher dose rocuronium can be also be used as an alternative to succinylcholine in a RSI. In any case, adequate muscle relaxation is paramount in order to prevent coughing or bucking on the endotracheal tube and the subsequent increase in ICP. Once the airway has been secured the primary goal is to maintain end organ perfusion pressure, with a CPP goal of greater than $60 \mathrm{mmHg}$ [10]. Intravascular volume should be maintained with the use of an isotonic intravenous fluid as needed. There is a potential benefit for hypertonic saline in patients with $\mathrm{TBI}$, as it may help to maintain intravascular volume with less cerebral edema. Hypotonic solutions such as Lactated Ringers should be avoided [11]. These patients may receive diuretics such as mannitol in order to treat cerebral edema, which may lead to further hypovolemia.

Intraoperatively, TBI patients should receive insulin if necessary to maintain euglycemia. Typically, an arterial line to acutely monitor blood pressure and two large bore intravenous catheters are recommended. In this patient population, higher levels of volatile anesthetics are routinely avoided because of the concern for cerebral vasodilation and increased ICP, leading to a decreased CPP. Maintenance of anesthesia may be maintained with intravenous agents, such as propofol, with the addition of vasopressors if needed to maintain MAP. In addition, although neurmonitoring is uncommon during emergent cases, somatosensory and/or motor evoked potentials may be used to access the integrity of the nervous system intraoperatively. If neuromonitoring is utilized, the anesthetic regimen should be adjusted accordingly. A balanced anesthetic technique or total intravenous anesthetic would be ideal in this instance. Electroencephalography may be used to detect seizures or guide titration of anesthetics if burst suppression is required. If seizures are detected, intravenous propofol or benzodiazepines may be administered. Anesthesiologists must always be prepared for massive hemorrhage in these cases, especially when major vessels are involved. Therefore, large-bore IV access and a type and cross for possible blood transfusion are crucial for management. TBI patients may develop coagulopathies such as disseminated intravascular coagulation rom the release of brain tissue thromboplastin into the circulation. If suspected, coagulopathic states should be monitored and aggressively treated. They may also acquire acute respiratory distress syndrome from the release of these mediators into the systemic circulation, necessitating the use of a pulmonary protective ventilation plan with positive end expiratory pressure and low tidal volumes intraoperatively. TBI patients may develop intraoperative complications such as seizures, significant hypotension following hematoma evacuation, cerebral ede$\mathrm{ma}$, and acute intracranial hemorrhage. The decision whether or not to consider extubation at the end of the surgical procedure depends on the degree of injury, the pre-operative neurological status, the extent of ICP elevation, the intraoperative course, and the nature of the patient's other concomitant injuries.

The majority of nail gun injuries are work-related accidents although deliberate self-inflicted injuries have become increasingly common [12]. The CT scan is critical to the initial assessment of PBI's, which can identify the entry point, object course, and a majority of acute initial injuries. If the object is in close proximity to major vascular structures, cerebral angiography may be useful to rule out acute vessel injury [13]. PBI's may cause arterial dissection, aneurysm or pseudoaneurysm formation, arterial or venous disruption, arterial-venous malformation, and bone fragmentation [14]. Although rare, traumatic intracranial aneurysms may have a delayed presentation of up to two to three weeks following the injury with a mortality of $50 \%$ following rupture [15]. Because of the potential for delayed detection of traumatic aneurysms, some authors advocate for angiography performed 7-14 days after the initial injury [16].

\section{Conclusion}

Although his initial injuries appeared ominous, this patient in this case was fortunate to not develop any permanent sequelae from his PBI's. Pneumatic nail gain injuries, though still uncommon, have increased in frequency over the past several decades. Successful management of these injuries requires a multi-disciplinary team approach to determine the safest intraoperative course. After the initial ATLS assessment, radiologic imaging utilizing $\mathrm{CT}$ and $\mathrm{CT}$ angiography is necessary to determine the extent of vascular injury and to determine the appropriate course of treatment. This case serves as a good example to highlight the anesthetic goals of TBI's and the subset of PBI's.

\section{References}

1. Faul M, Xu L, Wald MM, Coronado VG (2010) Traumatic brain injury in the United States: Emergency department visits, hospitalizations, and deaths. Centers for Disease Control and Prevention, National Center for Injury Prevention and Control.

2. Awori J, Wilkinson DA, Gemmete JJ, Thompson BG, Chaudhary N, et al. (2017) Penetrating head injury by a nail gun: case report, review of the literature, and management considerations. J Stroke Cerebrovasc Dis 26: e143-e149.

3. Centers for Disease Control and Prevention (2007) Nailgun injuries treated in emergency departments. United 
States MMWR Morb Mortal Wkly Rep 56: 329-332.

4. Vella MA, Crandall M, Patel MB (2017) Acute Management of Traumatic Brain Injury. Surg Clin North Am 97: 10151030.

5. Rudehill A, Bellander BM, Weitzberg E, Bredbacka S, Backheden M, et al. (2002) Outcome of traumatic brain injuries in 1,508 patients: Impact of prehospital care. J Neurotrauma 19: $855-868$.

6. Wang R, Li M, Gao WW, Guo Y, Chen J, et al. (2015) Outcomes of early decompressive craniectomy versus conventional medical management after severe traumatic brain injury: A systematic review and meta-analysis. Medicine (Baltimore) 94: e1733.

7. Sauvigny T, Göttsche J, Vettorazzi E, Westphal M, Regelsberger J (2016) New radiologic parameters predict clinical outcome after decompressive craniectomy. World Neurosurg 88: 519-525.

8. Schreckinger M, Orringer D, Thompson B, La Marca F, Sagher O, et al. (2011) Transorbital penetrating injury: Case series, review of the literature, and proposed management algorithm. J Neurosurg 114: 53-61.

9. Fan W, Zhu X, Wu L, Li D, Huang F, et al. (2015) Propofol: An anesthetic possessing neuroprotective effects. Eur Rev Med PharmacolSci 19: 1520-1529.
10. Griesdale DE, Örtenwall V, Norena M, Wong H, Sekhon MS, et al. (2015) Adherence to guidelines for management of cerebral perfusion pressure and outcome in patients who have severe traumatic brain injury. J Crit Care 30: 111-115.

11. Piper BJ, Harrigan PW (2015) Hypertonic saline in paediatric traumatic brain injury: A review of nine years' experience with $23.4 \%$ hypertonic saline as standard hyperosmolar therapy. Anaesth Intensive Care 43: 204-210.

12. Selvanathan S, Goldschlager T, McMillen J, Campbell S (2007) Penetrating craniocerebral injuries from nail-gun use. J Clin Neurosci 14: 678-683.

13. Springborg J, Eskesen V, Olsen N, Gjerris F (2007) Cranio-cerebral injuries caused by nail guns: Report on two cases, review of the literature and treatment algorithm. $J$ Trauma 63: E59-E64.

14. Luo W, Liu H, Hao S, Zhang Y, Li J, et al. (2012) Penetrating brain injury caused by nail guns: Two case reports and a review of the literature. Brain Inj 26: 1756-1762.

15. Blankenship B, Baxter A, McKahn G (1999) Delayed cerebral artery pseudoaneurysm after nail gun injury. AJR Am J Roentgenol 172: 541-542.

16. Litvack ZN, Hunt MA, Weinstein JS, West GA (2006) Self-inflicted nail-gun injury with 12 cranial penetrations and associated cerebral trauma. Case report and review of the literature. J Neurosurg 104: 828-834. 